\title{
Total parenteral nutrition in children and adolescents treated with high-dose chemotherapy followed by autologous haematopoietic transplants
}

\author{
Anna Wędrychowicz ${ }^{1,2} *$, Mikołaj Spodaryk ${ }^{3}$, Aleksandra Krasowska-Kwiecieńn ${ }^{1,2}$ and Jolanta Goździk ${ }^{1,2}$ \\ ${ }^{1}$ Transplantation Centre, University Children's Hospital, Cracow, Poland \\ ${ }^{2}$ Department of Transplantation, Polish-American Children's Hospital, Medical College, Jagiellonian University, \\ Wielicka Street 265, 30-663 Cracow, Poland \\ ${ }^{3}$ Department of Paediatrics, Gastroenterology and Nutrition, Polish-American Children's Hospital, Medical College, \\ Jagiellonian University, Wielicka Street 265, 30-663 Cracow, Poland
}

(Received 25 March 2009 - Revised 15 September 2009 - Accepted 16 September 2009 - First published online 28 October 2009)

Total parenteral nutrition (TPN) is still of great importance for haematopoietic stem cell transplantation (HSCT) patients because one of the major adverse effects of the high-dose therapy followed by HSCT is an inadequate oral nutrition intake. The aim of the study was analysis of TPN of young patients in the HSCT period. Twenty-two patients 1.8-20.8 year-old, median 5.4, treated with high-dose therapy and autologous HSCT because of malignancy were included into the study. Grafts contained $1.35-7.9 \times 10^{6}$, median $3.75 \times 10^{6} \mathrm{CD} 34^{+}$cells $/ \mathrm{kg}$. Engraftment occurred as follows: granulocytes $>0.5 \times 10^{9} / 1$ on $+11 \mathrm{~d}(8-25)$; platelets $>20 \times 10^{9} / 1$ on $+23 \mathrm{~d}(12-67)$. Patients were given isoenergetic, isonitrogenous TPN until they consumed less than $50 \%$ of their required diet orally. Proteins intake was $0 \cdot 8-2 \cdot 0 \mathrm{~g} / \mathrm{kg}$ per d, fats intake $1 \cdot 0-3 \cdot 0 \mathrm{~g} / \mathrm{kg}$ per d. Total non-proteins energies-nitrogen grams index was 140:1-200:1. Supplementation of electrolytes, microelements, trace elements and vitamins was dependent on individual patient requirement. TPN duration did not correlate with $\mathrm{CD} 34^{+}$cells number but correlated with platelets reconstitution. The assessment of nutritional condition demonstrated no differences in anthropometric parameters, but increase of serum albumin levels after TPN. Requirement for $\mathrm{P}^{3-}$ was above the normal ranges and correlated positively with platelets reconstitution. Requirement for $\mathrm{P}^{3-}$ and $\mathrm{K}^{+}$was higher in patients with mucositis than in other patients. Any complications due to TPN were observed. Adequately composed isoenergetic and isonitrogenous TPN with replacement of electrolytes according to their requirement in the early post-transplantation period allows not only improvement in nutritional status of patients but also could contribute to reconstitution of haematopoiesis.

Total parenteral nutrition: Autologous haematopoietic transplants: P intake: Haematopoiesis recovery

The treatment consisting of high-dose chemotherapy followed by autologous haematopoietic stem cell transplantation (HSCT) is mainly indicated in patients with malignant disease. Those patients have commonly experienced malnutrition associated with malignancy and its primary therapy. Additionally, HSCT is a highly stressful clinical situation as a consequence of cytoreductive therapy, infections or occurrence of other complications and tissue repairing process. One of the major adverse effects of the high-dose chemotherapy is an inadequate oral fluid and food intake that may result in dehydration and malnutrition. It is associated with mucositis that affects most of transplanted patients. Mucositis coincides with profound changes in the integrity of the mucosal epithelia that line the oral cavity, oesophagus and gastrointestinal tract due to the effects of high-dose chemotherapy on cells with high turnover rates. These changes result in a denuded mucosa, which can lead to bacterial, viral or fungal invasion of the bowel wall, sepsis, ulceration, bleeding, malabsorption, diarrhoea and pain throughout the gastrointestinal tract ${ }^{(1)}$. Adequate nutrition support during HSCT according to the patient's gastrointestinal function is an important principle of this combined therapy. There were different experiences in nutritional support, total parenteral nutrition (TPN), partial PN (PPN) or only enteral nutrition ${ }^{(2-5)}$. Lately, there have been some experiments with intensive enteral nutrition support but without spectacular effects so PN is still of great importance in transplantation period. Sefcick et al. ${ }^{(4)}$ carried out a pilot study of fifteen adult allogeneic patients with elective naso-jejunal placements before conditioning chemotherapy. Although the authors deemed the study successful, patients lost $4.5 \%$ of body weight at the time of discharge and experienced profound difficulties with tube maintenance due to vomiting and reported epistaxis. They also experienced difficulties with feeding tolerance owing to diarrhoea and discomfort. Enteral studies in transplanted patients are fraught with difficulty as evidenced by the Sefcick study, but also due to the confusion between enteral intolerance and side effects of high-dose chemotherapy, in the face of mucositis and thrombocytopenia. Additionally, enteral feeding is often combined with PPN, resulting in an unclear role of enteral nutrition.

Abbreviations: BMT, bone marrow transplantation; HSCT, haematopoietic stem cell transplantation; PPN, partial parenteral nutrition; TPN, total PN.

* Corresponding author: Dr Anna Wędrychowicz, fax +48 12 6591594, email miniedzw@cyf-kr.edu.pl 
Besides, TPN is easier to administer because transplant patients are required to have central venous access for the whole transplantation procedure. TPN has been and continues to be the mainstay of nutrition support for HSCT patients because it is easy to administer, it is provided under the assumption that it is beneficial and preferred by transplant physician $^{(6)}$. There are some voices that PN should be reserved as second-line therapy for those patients who are unable to tolerate enteral feedings ${ }^{(7)}$. The same authors postulate that TPN should not be given to patients only in order to maintain their nutritional status during the peritransplant period. These postulates are worth discussing but not in paediatric patients. In this group of patients, normal development and maturation are one of the main goals of the whole therapy. Additionally complete collaboration with a sick child is often impossible. The changes of TPN methods in transplanted patients have been still observed, which serve to gain optimal results of the whole therapy.

A retrospective analysis of TPN in twenty-two patients treated with high-dose chemotherapy and autologous HSCT in Transplantation Centre of University Children's Hospital in Cracow was aimed to provide an optimal method of nutritional support in the period of transplantation.

\section{Materials and methods}

There were twenty-two patients: eleven girls and eleven boys in the age of $1.8-20.8$ years, median 5.4 years included into the study. High-dose chemotherapy and autologous haematopoietic stem cells transplantation were indicated because of: neuroblastoma in fourteen patients; Ewing's sarcoma in four patients; Hodgkin's disease in two patients; yolk sac tumour in one; acute lymphoblastic leukaemia also in one case.

\section{High-dose chemotherapy and autologous haematopoietic stem} cells transplantation

High-dose chemotherapy consisted of busulphan and melphalan in ten patients, cyclophosphamide, etoposide and melphalan in nine patients, bis-chloronitrosourea (BCNU), etoposide, ara-C and melphalan in two patients and cyklofosfamid and total body irradiation in one patient. The median number of transplanted $\mathrm{CD} 34^{+}$cells was $3.75 \times 10^{6}$ cells $/ \mathrm{kg}$ (range $1 \cdot 35-7 \cdot 9$ ). All our patients were hospitalised in rooms with laminar airflow. High efficiency particulate air filtration with positive pressure was used. Red cells and platelet concentrates were used according to the transfusion policy recommended by European Group for Blood and Marrow Transplantation. Since day +5 we administered granulocyte colony-stimulating factor in a daily dose of $5 \mu \mathrm{g} / \mathrm{kg}$.

\section{Nutritional treatment}

Patients were given isoenergetic, isonitrogenous TPN when they had consumed less than $50 \%$ of their required diet orally for previous $2 \mathrm{~d}$. If patients could be nourished orally in the period of neutropenia, they received sterile, boiled diet, without gluten and lactose. After engraftment time, they received low-bacterial diet. We continued TPN until they consumed $50 \%$ of their required diet orally again. TPN was administered through central venous line (Hickman or
Broviac catheters or vascuports). Volumes of TPN depended on patients' body mass, and were corrected daily according to additional venous fluid administration and fluid loss. In some cases, we constituted nutritional mixture according to individual protocol because of fluid restriction necessity. Usually we used standard basic fluid that contained $2 \%$ of aminoacids and $12 \%$ of glucose with total non-protein energies-nitrogen ratio $127 \cdot 5: 1$, which was supplemented with fat emulsion to an optimal value 140:1-200:1. This ratio was dependent on individual clinical assessment, which included nutritional status using anthropometric and biochemical methods. According to the age and the duration of TPN, the protein intake was $0.8-1.5 \mathrm{~g} / \mathrm{kg}$ per $\mathrm{d}$ and the fat intake was $1.0-3.0 \mathrm{~g} / \mathrm{kg}$ per d. Supplementation of electrolytes, microelements, trace elements and vitamins was dependent on individual patient requirement.

During TPN, our patients were monitored daily by our team. Fluid intake and output as well as central venous catheter site and function were monitored continuously. Blood glucose, weight and electrolytes were monitored twice a day, when the patient became stable blood tests were done once a day ( $\mathrm{Na}, \mathrm{K}, \mathrm{Cl}, \mathrm{Ca}, \mathrm{Mg}$ and $\mathrm{P}$ ). Blood cell count was measured daily. Liver function tests, bilirubin, prothrombin time, cholesterol, TAG, total protein, albumin, C-reactive protein, creatinine and urea were measured twice a week. All the above-mentioned parameters were measured also initially before TPN administration.

\section{Biochemical measurements}

Electrolytes, liver function tests, prothrombin time, total protein, albumin, C-reactive protein, creatinine and urea were measured by dry biochemistry method using Vitros 950 (Ortho Clinical Diagnostics, Rochester, NY, USA). IgA was measured by nephelometric method (Behring nephelometric II; Dade Behring, Newark, DE, USA). Prothrombin time was measured by Quick method using Sysmex CA-500 (Dade Behring). Cholesterol and TAG were measured with enzymatic method (Randox kit) using Cobas-Bio.

\section{Statistical analysis}

Statistical calculations were performed using the Statistica program (StatSoft, Inc., Tulsa, OK, USA). Results are expressed as medians and ranges. The Mann-Whitney $U$ test and Spearman correlation were used for statistical analysis, $P<0.05$ was considered statistically significant.

\section{Results}

\section{Clinical outcome and nutritional status}

Patients continued TPN for median 19d (range 8-35), from day 0 to +35 . Mucositis was observed in eighteen $(82 \%)$ of our patients and was the most frequent toxicity of this treatment. Engraftment occurred as follows: median time to reach granulocytes $>0.5 \times 10^{9}$ per litre was $11 \mathrm{~d}$ (range $8-25$ ); leukocytes $>1.0 \times 10^{9}$ per litre was $10 \mathrm{~d}$ (range 9-25). For platelets $>20 \times 10^{9}$ per litre, it was $23 \mathrm{~d}$ (range 12-67) and for platelets $>50 \times 10^{9}$ per litre, it was $39 \mathrm{~d}$ (range 13-70). 


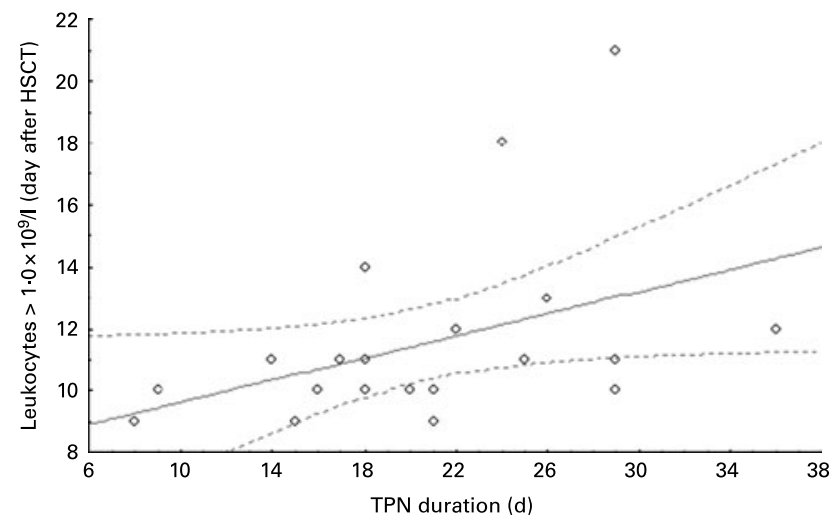

Fig. 1. Correlation between total parenteral nutrition (TPN) duration and leukocytes recovery in paediatric patients after autologous haematopoietic stem cells transplantation (HSCT). Correlation $r 0.42, P=0.05 .-\diamond-$, Regression $95 \% \mathrm{Cl}$.

The duration of TPN neither correlated with the number of transplanted $\mathrm{CD}_{3} 4^{+}$cells nor with dosage and duration of granulocyte colony-stimulating factor administration. But it correlated with the reconstitution of leukocytes $>1.0 \times 10^{9}$ per litre $(P=0.05 ; r 0.42)$ and platelets $>20 \times 10^{9}$ per litre $(P=0.04 ; r 0.45)$, Figs. 1 and 2.

Statistically longer TPN was received by patients with mucositis $(P=0.04)$ than without mucositis (four patients $18 \%)$, Fig. 3. In the patients with mucositis, we observed delayed haematopoiesis reconstitution $(P=0 \cdot 01)$, Fig. 4 . We also observed significantly delayed reconstitution of platelets in the patients with parasitic infections (three children with toxocariasis and one with ascardiasis). Patients with parasitic infections received TPN longer (median $23 \mathrm{~d}$ ) than others (median $18 \mathrm{~d}$ ) with statistical significance $(P=0 \cdot 02)$.

The body mass of patients estimated before TPN administration was about $90 \%$ of body mass appropriate for their height, so they had no malnutrition. When we assessed their nutritional condition after TPN, we did not observe any differences in anthropometric parameters, but we noticed significant increase of serum albumin levels $(P=0.0005)$, Fig. 5 .

There were no side effects due to TPN: technical problems with central catheters; infections associated with TPN or metabolic complications such as significant increase of lipids,

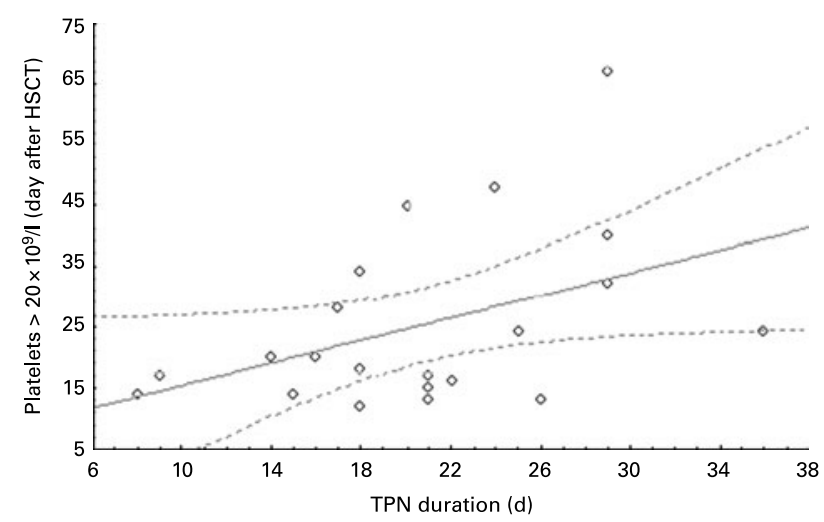

Fig. 2. Correlation between total parenteral nutrition (TPN) duration and platelets recovery in paediatric patients after autologous haematopoietic stem cells transplantation (HSCT). Correlation $r 0.44, P=0.04 .-\diamond-$, Regression $95 \% \mathrm{Cl}$.

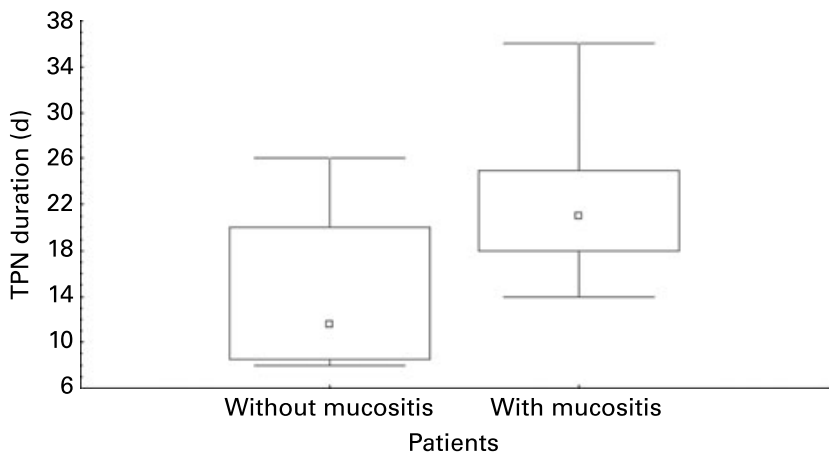

Fig. 3. Duration of total parenteral nutrition (TPN) in paediatric patients with and without mucositis after autologous haematopoietic stem cells transplantation. $P=0.41$.

glucose and liver function tests level (Table 1); disturbances of electrolytes concentration; statistically significant patient body mass gain.

\section{Requirement for electrolytes}

The median requirement for $\mathrm{K}^{+}$in our patients amounted $3.0 \mathrm{mEq} / \mathrm{kg}$ per $\mathrm{d}$ (range $1.09-5.44$ ) and was within normal range(s) (the reference ranges $1-4 \mathrm{mEq} / \mathrm{kg}$ per $\mathrm{d}^{(8)}$ ). For $\mathrm{Ca}^{2+}$, it was median $0.29 \mathrm{mEq} / \mathrm{kg}$ per d (range 0.07-0.99; the reference ranges $0.5-3.0 \mathrm{mEq} / \mathrm{kg}$ per $\left.\mathrm{d}^{(8)}\right)$ and for $\mathrm{Mg}^{2+}$, median $0.3 \mathrm{mEq} / \mathrm{kg}$ per $\mathrm{d}$ (range $0.08-1.01$ ) and was below norms (the reference ranges $3 \cdot 0-5.0 \mathrm{mEq} / \mathrm{kg}$ per $\mathrm{d}^{(8)}$ ). We observed requirement for $\mathrm{P}^{3-}$ above the normal ranges $\left(0.5-1.0 \mathrm{mEq} / \mathrm{kg}\right.$ per $\left.\mathrm{d}^{(8)}\right)$. The median was $1.1 \mathrm{mEq} / \mathrm{kg}$ per d (range 0-2.4).

Patients with mucositis had significantly higher requirement for $\mathrm{P}^{3-}(P=0.004)$ and $\mathrm{K}^{+}(P=0.003)$ than patients who had no(t) mucositis, Figs. 6 and 7.

Requirement for electrolytes neither correlated with TPN duration, the number of transplanted $\mathrm{CD} 34^{+}$cells nor the dosage and duration of G-CSF administration. We did not find significant correlation between requirement for $\mathrm{K}^{+}$and $\mathrm{Ca}^{2+}$ and haematopoiesis reconstitution indicators. However, requirement for $\mathrm{Mg}^{2+}$ correlated negatively with reconstitution of leukocytes $>1.0 \times 10^{9}$ per litre $(P=0.02 ; r-0.5)$ and platelets $>20 \times 10^{9}$ per litre $(P=0 \cdot 01 ; r-0 \cdot 54)$. Requirement for $\mathrm{P}^{3-}$ correlated positively with reconstitution of platelets $>50 \times 10^{9} / 1(P=0 \cdot 02 ; r 0 \cdot 54)$, Fig. 8 .

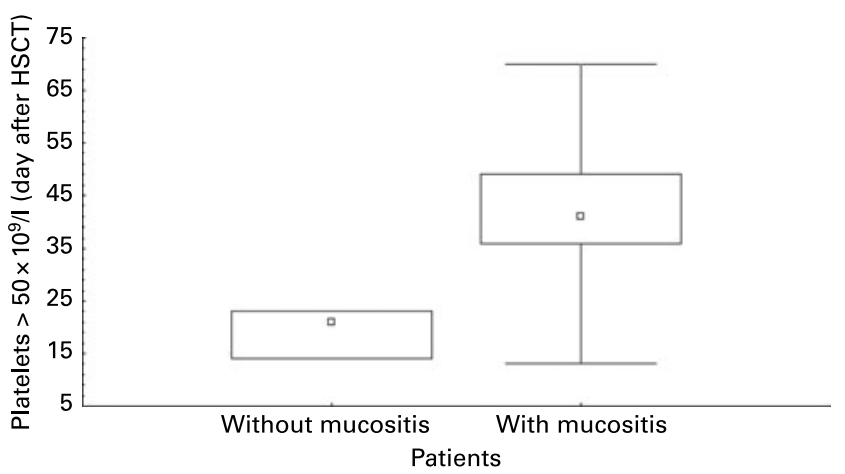

Fig. 4. Platelets reconstitution in paediatric patients with and without mucositis after autologous haematopoietic stem cells transplantation (HSCT) supported with total parenteral nutrition. $P=0.012$. 


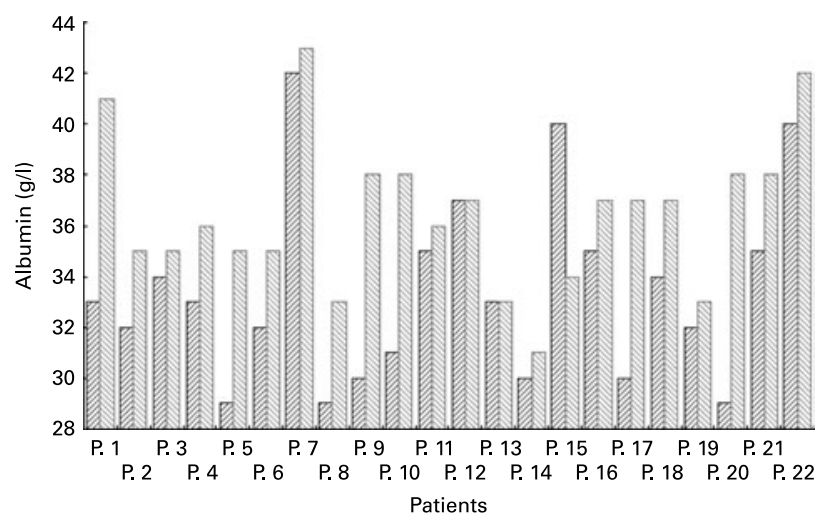

Fig. 5. Serum albumin levels of paediatric patients after autologous haematopoietic stem cells transplantation before and at the end of total parenteral nutrition administration. Albumin start; $\mathbb{\mathbb { Q }}$, albumin stop.

\section{Discussion}

Different methods of nutritional support are used in the early period after haematopoietic stem cells transplantation ${ }^{(3,9-11)}$. Although nowadays intensive experience with enteral feeding is provided in transplantation centres ${ }^{(4,12)}$, PN has still been considered the method of choice for paediatric patients undergoing bone marrow transplantation $(\mathrm{BMT})^{(5)}$.

There exists some kind of prophylaxis when children are given TPN until they consume $50 \%$ of their diet orally. Our patients were given isoenergies and isonitrogenous $\mathrm{PN}$ in such conditions. We did not observe malnutrition in the patients at the beginning of the treatment. Their body mass was about $90 \%$ of body mass appropriate for their height. But a biochemical marker as the level of albumin indicated impairment of nutritional status during the course of highdose chemotherapy before TPN administration. At the end of PN support we did not observe significant differences in body mass but albumin levels increased and normalised. Simultaneously, the assessment of inflammatory status before and at the end of TPN administration could exclude the possibility of influence of inflammation status on serum albumin level (Table 1). Also in the study of Hwang et al., patients receiving TPN improved their nutrition status and increased their body mass compared to those receiving PPN.
The result of PPN was also a decrease of patients' visceral proteins which has not been observed in patients who received $\mathrm{TPN}^{(9)}$. In another study, the marked variations in anthropometric parameters and albumin levels were not observed in children who were given TPN. However, prealbumin and retinol-binding protein showed statistically significant, positive variations as the response to nutritional repletion ${ }^{(13)}$. We did not measure these markers. It was recently reported than in critically ill patients, the serum prealbumin level did not respond sensitively to nutritional support. In addition, an increase in the prealbumin level was not the indicator of better prognosis for these groups of patients ${ }^{(14)}$. As opposed to prealbumin, albumin level is the well-recognised biochemical marker of nutritional status in different metabolic states $^{(15,16)}$

There were some experiences to answer the question of what is the appropriate energy level in TPN during the period of transplantation. Following the old recommendations, the energy intake with TPN in the early time after HSCT in children should be 1.5-1.7 BMR with $1.5 \mathrm{~g}$ of protein $/ \mathrm{kg}$ per d. According to the study of Yokoyama et $a l .{ }^{(17)}$, the predicted energy requirement to maintain body weight after HSCT would be $128 \%$ of basal energy expenditure. But it was reported that delivering TPN with reduced parenteral energy and protein intake after BMT appeared to minimise $\mathrm{Na}^{+}$and $\mathrm{K}^{+}$disturbances and improve serum albumin concentrations without having any adverse effect on nitrogen balance ${ }^{(18)}$. The study of Forchielli et al. ${ }^{(5)}$, also has shown that the energy supplied to children with TPN in BMT is less than expected. Their patients received $72 \%$ prescribed energies that were 0.87 BMR and mean 176:1 non-protein energies:nitrogen ratio. Similarly, our data suggest that isoenergetic TPN is an effective method of PN, which prevents malnutrition, maintains body weight and improve nutritional status of the patients early after autologous HSCT.

The same problem like appropriate energy level regards intake of proteins and lipid components in nutritional emulsion. Hypoproteinaemia has been reported in patients after BMT probably due to: negative nitrogen balance; increased catabolism; fluid redistribution; decreased protein synthesis, resulting from cytotoxic chemotherapy; reduced nutrient intake.

Table 1. Body mass and some biochemical and immunological parameters of patients treated with high-dose chemotherapy and autologous haematopoietic stem cells transplantation before and at the last day of total parenteral nutrition (TPN) administration

(Median values and ranges)

\begin{tabular}{lcccccc}
\hline & \multicolumn{2}{c}{ First day of TPN } & & \multicolumn{2}{c}{ Last day of TPN } & \\
\cline { 2 - 3 } & Range & Median & & Range & Median & $P$ \\
\hline Body mass $(\mathrm{kg})$ & $9-54$ & 16.6 & & $9.1-54.5$ & 16.1 & 0.81 \\
Albumin $(\mathrm{g} / \mathrm{l})$ & $29-42$ & 33 & & $31-43$ & 36.5 & 0.0005 \\
Glucose $(\mathrm{mmol} / \mathrm{l})$ & $4.0-6.7$ & 5.1 & & $3.8-6.4$ & 4.9 & 0.06 \\
Chol (mmol/l) & $2.9-3.78$ & 2.94 & & $2.76-2.83$ & 2.8 & 0.2 \\
TAG (mmol/l) & $0.71-0.8$ & 0.76 & $0.79-1.02$ & 0.84 & 0.35 \\
ALT (U/l) & $16-303$ & 57 & & $18-123$ & 35 & 0.03 \\
CRP (mg/l) & $<3-171$ & $<3$ & & $<3-41.7$ & $<3$ & 0.97 \\
IgA (g/l) & $0.38-3.74$ & 1.21 & $0.28-1.16$ & 0.66 & 0.13 \\
\hline
\end{tabular}

Chol, cholesterol; ALT, alanine transaminase; CRP, C-reactive protein. 


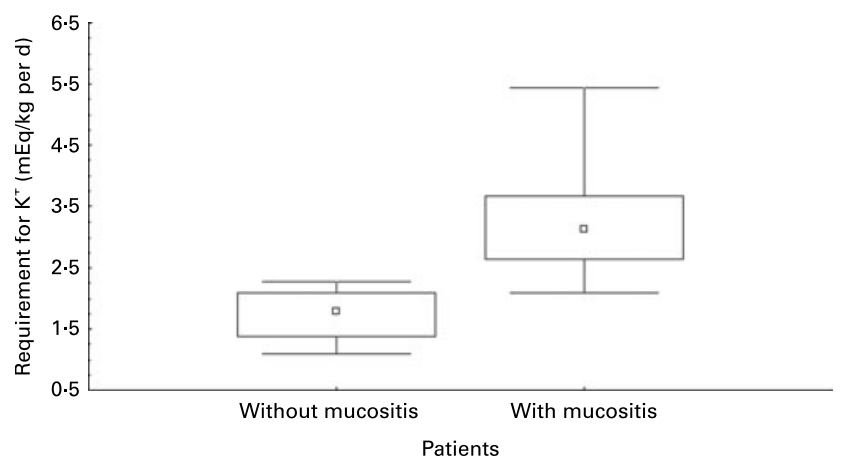

Fig. 6. Requirement for $\mathrm{K}^{+}$in paediatric patients with and without mucositis after autologous haematopoietic stem cells transplantation supported with total parenteral nutrition. $P=0.003$.

Thus, there were the same experiences with increasing nitrogen dose but without spectacular clinical metabolic effects $^{(19)}$. Simultaneously, it was reported that reducing parenteral protein intake improves metabolic homeostasis after BMT and increases serum albumin concentration ${ }^{(18)}$. The next question is how the quality of energy substrates administered with TPN after high-dose therapy influences the clinical outcome of patients undergoing BMT. It was presented that lipid-based TPN (80\% lipid, using an $n-6$ long-chain TAG emulsion and $20 \%$ glucose) resulted in significantly lower hyperglycaemia than in glucose-based (100\% glucose) $\mathrm{TPN}^{(11)}$. We did not observe significant hyperglycaemia in our patients who were given TPN always with lipids as about $30 \%$ of the energy source. Additionally, intravenously administered lipids might modulate the immune response and synthesis of cytokines, PG and leukotrienes. It was reported that probably in this way lipids could participate in the pathogenesis of graft $v$. host disease after allogeneic stem cells transplantation ${ }^{(11)}$.

Good nutrition for children is considered as immunonutrition, and we did not use the immunomodulating nutrients like arginine, glutamine, $n$-3-fatty acids and nucleotides. Paediatric experience in this subject is limited ${ }^{(20)}$. Opinions on TPN supplemented with glutamine in BMT period are different. Ziegler et al. ${ }^{(21)}$ reported its benefits but in the study of Schloerb \& Amare ${ }^{(22)}$, substitution of glutamine during TPN did not have discernible clinical or laboratory effects. But other authors suggested that immunonutrition

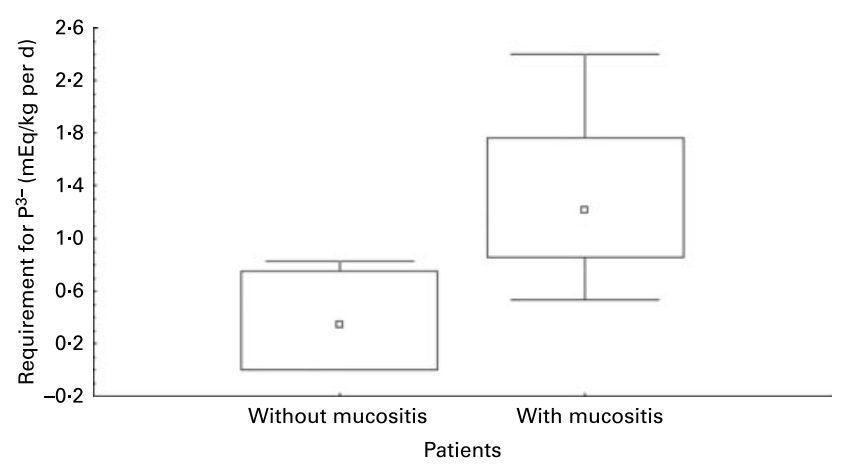

Fig. 7. Requirement for $\mathrm{P}^{3-}$ in paediatric patients with and without mucositis after autologous haematopoietic stem cells transplantation supported with total parenteral nutrition. $P=0.004$.

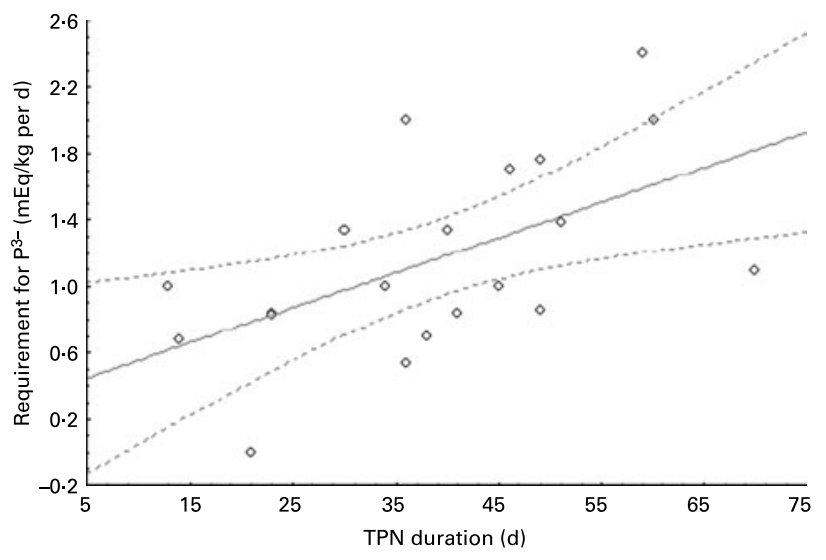

Fig. 8. Correlation between requirement for $\mathrm{P}^{3-}$ and platelets reconstitution in paediatric patients after autologous haematopoietic stem cells transplantation during total parenteral nutrition (TPN) support. Correlation $r 0.54$, $P=0.02 .-\diamond-$, Regression $95 \% \mathrm{Cl}$.

may provide a less invasive alternative to immunotherapy in protecting against cancer associated with chronic inflammation ${ }^{(23)}$. There was also an opinion that glutamine supplementation cannot be recommended to all HSCT recipients because it has been shown to increase morbidity and mortality rates in autologous transplant patients ${ }^{(7)}$. Glutamine is highly used by rapidly dividing cells such as blood cells and those in the gastrointestinal tract, but also neoplastic cells. Glutamine is necessary for the in vitro growth and function of $\mathrm{T}$ lymphocytes and natural killer cells. Because glutamine is the main source of nitrogen for tumour cells, it could have the deleterious effect of feeding the tumour. The presence of a tumour produces great changes in host glutamine metabolism. Because glutamine depletion has adverse effects for the host, the effect of glutamine supplementation in the tumour-bearing state could bring positive results for the host ${ }^{(24)}$. It was presented that supplemental oral glutamine reduced tumour growth in experimental breast cancer ${ }^{(25)}$. In implantable sarcoma and breast carcinoma rat models, it was demonstrated that daily oral administration of glutamine results in a $40 \%$ decrease in tumour growth compared to isonitrogenous-fed controls ${ }^{(26)}$. Moreover, the data of some studies allow suggestion that dietary supplemental glutamine could be used in the clinical practice to increase the therapeutic index of cancer treatments by protecting normal tissues from, and sensitising tumour cells to, chemotherapy and radiation-related injury ${ }^{(27,28)}$. Enhancing the antioxidant status of the body and activation of apoptosis exerts chemopreventive effects of glutamine ${ }^{(29)}$. On the other hand, the double-blind, randomised study on glutamine supplementation in cancer patients receiving chemotherapy concluded that glutamine did not have a significant effect on either tumour response or secondary effects of chemotherapy ${ }^{(30)}$. So, until more information is available, nutritional support should focus primarily on preventing nutritional deficiencies rather than on immunomodulation ${ }^{(31)}$. Results of the largest randomised, controlled trial found that in the general population treated in intensive care units, immunonutrition has no beneficial effect on clinical outcome parameters ${ }^{(32)}$. Glutamine helps maintain normal secretory, $\operatorname{IgA}$ an immune substance in the gut ${ }^{(33)}$. There were no statistical differences in serum $\operatorname{IgA}$ level in 
our patients before TPN and after it, although there was a tendency to decrease it (Table 1). We did not supply glutamine in TPN support. Of course, serum IgA is not the same as mucosal $\operatorname{IgA}$, but it could indirectly indicate that glutamine deficiency in our patients is not so significant even in spite of it that most of them had mucositis. The catabolic state and immunological disorders caused by high-dose chemoradiotherapy and autologous HSCT are relationally not so long. In our opinion, it would be very interesting to study the role of dietary supplemental glutamine in the patients after allogeneic HSCT, especially in the group with severe mucositis and intestinal form of graft $v$. host reaction.

In our group, TPN was given for 8-35d, median $19 \mathrm{~d}$. In the literature ${ }^{(5,10,34)}$, the time of TPN administration in the early period after BMT was longer than in our group, which of course was associated with greater costs of whole support. Children with mucositis received TPN statistically longer than patients without this toxicity of high-dose chemotherapy. Of course, a small number of patients without mucositis do not allow a confident comparison, but data obtained in the study seem to be very probable. In the group with mucositis, the requirement for $\mathrm{P}$ and $\mathrm{K}$ was higher than in the wholepresented group. Clinical symptoms of mucositis such as pain, nausea, vomiting and in the same case diarrhoea are connected with the intestinal depletion of $\mathrm{K}$. It could be one of the reasons of the higher requirement for $\mathrm{K}^{+}$in the patients with mucositis. The requirement for $\mathrm{P}$ was higher not only in the patients with mucositis but also in all our patients. Engraftment associated hypophosphataemia has been infrequently reported ${ }^{(35-37)}$. The possible mechanisms of it are both a direct effect of cytokine release and an increased consumption by the dividing cells ${ }^{(38)}$. Patients with mucositis could additionally have high phosphate uptake by the replicating mucosa cells.

Among other complications, only parasitic infections were associated with longer than mean TPN duration. Three out of the presented twenty-two patients $(13.6 \%)$ had toxocariasis ${ }^{(39)}$ and one child had ascaridiasis. In these patients, besides longer TPN administration, we also observed delayed platelet reconstitution. It could have been caused indirectly by increased catabolism due to infection or directly by antiparasitic drugs but also by additional amount of antigens released from destroyed larvae. Association between thrombopoiesis and toxocariasis has been already reported by Kagialis-Girard et al. ${ }^{(40)}$, who described two cases of toxocariasis with persistent secondary thrombocytosis.

Recovery of haematopoiesis in our patients was within normal ranges in comparison to data from the literature. It was reported that engraftment of donor marrow cells was $3 \mathrm{~d}$ earlier in the patients received TPN group than in controls who received nutritional support ad libitum after $\mathrm{BMT}^{(34)}$. In opposite, Cetin et al. ${ }^{(41)}$, reported that autologous BMT patients receiving TPN had longer delays in platelet engraftment than patients receiving PPN. But implications of their findings are limited due to the non-random design, failure to document similarities between groups for medical and nutritional status at the time of admission and exposure of all participants to PN. The present study showed that mucositis significantly prolonged haematopoesis recovery. Thus, no wonder that patients treated with TPN because of severe mucositis received TPN longer than patients with lower grade mucositis treated with PPN. Later, study of Weisdorf did not show the effect of TPN on engraftment but significantly influenced long-term outcome of BMT. So, in spite of the cost, the prophylactic nutritional therapy appears to be beneficial even for well-nourished individuals during cytoreduction and $\mathrm{BMT}^{(42)}$.

TPN often takes place without complications. We did not observe any complications due to TPN: technical problems with central catheters; infections or metabolic complications such significant increase of lipid, glucose and liver function tests level; disturbances of electrolytes concentration; statistically significant patient body mass gain. In our opinion, infectious complications can be eliminated with adequate care of central venous catheter. Data presented by Uderzo ${ }^{(13)}$ showed that TPN was not responsible for infection in any patient. They had mild and easily controlled metabolic complications. In the study of Forchielli et al. ${ }^{(5)}$, infections and temporarily increased liver function tests have been observed in patients given TPN during BMT. On the other hand, compared to TPN, the PPN/enteral nutrition group showed a twofold number of patients with a positive blood culture, but this observation did not reach statistical significance $^{(43)}$. Sheean \& Braunschweig showed significant decrease in oral intake patterns and a tendency toward infections for autologous and allogeneic PN $v$. non-PN recipients. However, simultaneously significantly more mucositis was observed on days before $\mathrm{PN}$ initiation in $\mathrm{PN} v$. non-PN patients $^{(44)}$. So probably mucositis, and not $\mathrm{PN}$, was responsible for the decrease in oral intake patterns and the tendency towards infections.

\section{Conclusion}

Isoenergetic and isonitrogenous TPN with adequate supplementation of electrolytes, microelements, trace elements and vitamins is a safety and often the only possible method of nutritional treatment in the early time after autologous HSCT in paediatric patients. It allows to improve their nutritional status and contribute to reconstitution of haematopoiesis. Occurrence of mucositis in patients treated with high-dose chemotherapy and autologous haematopoietic stem cells transplantation significantly prolonged the duration of TPN administration but also delayed haematopoiesis reconstitution process that is associated with increased requirement for $\mathrm{P}$. It is closely associated with increased requirement for $\mathrm{K}$ through its intestinal depletion and also increased requirement for $\mathrm{P}$ taken to haematopoiesis reconstruction.

The present study on TPN in patients after HSCT showed positive effects on their nutritional state and the specific requirements for electrolytes especially in the case of coexistent mucositis. Our findings confirm the crucial effect of nutrition on chronic diseases, underlying the cases of increased catabolism induced by specific treatments.

\section{Acknowledgements}

This research received no specific grant from any funding agency in the public, commercial or not-for-profit sectors. There are no conflicts of interest. A. W., A. K.-K. and M. S. administered TPN in our patients. A. W. analysed the 
nutritional support in transplanted children and adolescents. M. S. and A. W. coordinated and provided nutritional support in our department. J. G. is clinical program director of our transplant team.

\section{References}

1. Rapoport AP, Miller LF, Linder T, et al. (1999) Analysis of factors that correlate with mucositis in recipients of autologous and allogeneic stem cell transplants. J Clin Onc 17, 2446-2453.

2. Szeluga DJ, Stuart RK, Brookmeyer R, et al. (1987) Nutritional support of bone marrow transplant recipients: a prospective, randomized clinical trial comparing total parental nutrition to an enteral feeding program. Cancer Res 47, 3309-3316.

3. Gomez Candela C, de Cos Blanco AI, Martinez Olmos MA, et al. (1997) Nutritional support in bone marrow transplantation. Nutr Hosp 12, 263-269.

4. Sefcick A, Anderton D, Byrne JL, et al. (2001) Naso-jejunal feeding allogeneic bone marrow transplant recipients: results of pilot study. Bone Marrow Transplant 28, 1135-1139.

5. Forchielli ML, Azzi N, Cadranel S, et al. (2003) Total parental nutrition in bone marrow transplant: what is the appropriate energy level? Oncology 64, 7-13.

6. Sheean PM (2005) Nutrition support of blood or marrow transplant recipients: how much do we really know? Pract Gastroenterol 4, 84-97.

7. Afrons LM \& Lazarus HM (2005) Total parenteral nutrition and hematopoietic stem cell transplantation: an expensive placebo? Bone Marrow Transplant 36, 281-288.

8. Spodaryk M (2001) Nutritional Support of Children. Cracow: Jagiellonian University Publications.

9. McDiarmid S (2002) Nutritional support of the patient receiving high-dose therapy with hematopoietic stem cell support. Can Oncol Nurs $J$ 12, 102-115.

10. Hwang TL, Chiang CL \& Wang PN (2001) Parental nutrition support after bone marrow transplantation: comparison of total and partial parental nutrition during the early posttransplantation period. Nutrition 17, 773-775.

11. Muscaritoli M, Conversano L, Torelli GF, et al. (1998) Clinical and metabolic effects of different parenteral nutrition regiments in patients undergoing allogeneic bone marrow transplantation. Transplantation 66, 610-616.

12. Langdana A, Tully N, Molloy E, et al. (2001) Intensive enteral nutrition support in pediatric bone marrow. Bone Marrow Transplant 27, 741-746.

13. Uderzo C, Rovelli A, Bonomi M, et al. (1991) Total parental nutrition and nutritional assessment and leukaemic children undergoing bone marrow transplantation. Eur J Cancer 27, $758-762$.

14. Lim SH, Lee JS, Chae SH, et al. (2005) Prealbumin is not sensitive indicator of nutrition and prognosis in critical ill patients. Yonsei Med J 46, 21-26.

15. Acosta Escribano J, Gomez-Tello V, Ruiz Santana S, et al. (2005) Nutritional assessment of the severely ill patient. Nutr Hosp 20, 5-8.

16. Ignacio de Ulibarri J, Gonzalez-Madrono A, de Villar NG, et al. (2005) CONUT: a tool for controlling nutritional status. First validation in a hospital population. Nutr Hosp 20, $38-45$.

17. Yokoyama S, Fujimoto T, Mitomi T, et al. (1989) Use of total parental nutrition in pediatric bone marrow transplantation. Nutrition 5, 27-30.

18. Taverhoff A, McArdle AH \& Rybka WB (1991) Reducing parenteral energy and protein intake improves metabolic homeostasis after bone marrow transplantation. Am $\mathrm{J}$ Clin Nutr 54, 1087-1092.

19. Geibig CB, Owens JP, Mirtallo JM, et al. (1991) Parental nutrition for marrow transplant recipients: evaluation of an increased nitrogen dose. JPEN J Parenter Enteral Nutr 15, $184-188$.

20. Singh R, Gopalan S \& Sibal A (2002) Immunonutrition. Indian J Pediatr 69, 417-419.

21. Ziegler TR, Young LS, Benfell K, et al. (1992) Clinical and metabolic efficacy of glutamine-supplemented parenteral nutrition after bone marrow transplantation. A randomized, doubleblind, controlled study. Ann Intern Med 116, 821-828.

22. Schloerb PR \& Amare M (1993) Total parental nutrition with glutamine in bone marrow transplantation and other clinical applications (a randomized, double-blind study). JPEN $J$ Parenter Enteral Nutr 17, 407-413.

23. Philpott M \& Ferguson LR (2004) Immunonutrition and cancer. Mutat Res 551, 29-42.

24. Medine MA (2001) Glutamine and cancer. J Nutr 131, 2539S-2542S.

25. Todorova VK, Harms SA, Kaufmann Y, et al. (2004) Effect of dietary glutamine on tumor gluthatione levels and apoptosisrelated proteins in DMBA-induced breast cancer of rats. Breast Cancer Res Treat 88, 247-256.

26. Klimberg VS (2005) Is glutamine effective in enhancing host immune response to tumors? J Nutr 135, 2920S.

27. Kaufmann Y, Todorova VK, Luo S, et al. (2008) Glutamine affects glutathione recycling enzymes in a DMBA-induced breast cancer model. Nutr Cancer 60, 518-525.

28. Todorova V, Vanderpool D, Blossom S, et al. (2009) Oral glutamine protects against cyclophosphamide-induced cardiotoxicity in experimental rats through increase of cardiac glutathione. Nutrition 25, 812-817.

29. Lim V, Korourian S, Todorova VK, et al. (2009) Glutamine prevents DMBA-induced squamous cell cancer. Oral Oncol $\mathbf{4 5}$ $148-155$

30. Bozzetti F, Biganzoli L, Gavazzi C, et al. (1997) Glutamine supplementation in cancer patients receiving chemotherapy: a double-blind randomized study. Nutrition 13, 748-751.

31. Stechmiller JK, Childress B, Porter T, et al. (2004) Arginine immunonutrition in critically ill patients: a clinical dilemma. Am J Crit Care 13, 17-23.

32. Kieft H, Roos AN, van Drunen JD, et al. (2005) Clinical outcome of immunonutrition in a heterogeneous intensive care population. Intensive Care Med 31, 501-503.

33. Kudsk KA, Wu Y, Fukatsu K, et al. (2000) Glutamine-enriched total parenteral nutrition maintains intestinal interleukin-4 and mucosal immunoglobulin A levels. JPEN J Parenter Enteral Nutr 24, 270-275.

34. Weisdorf S, Hofland C, Sharp HL, et al. (1984) Total parental nutrition in bone marrow transplantation: a clinical evaluation. J Peadiatr Gastroenterol Nutr 3, 95-100.

35. Raanani P, Berkowicz M, Harden I, et al. (1995) Severe hypophosphataemia in autograft recipients during accelerated leukocyte recovery. Br J Haematol 91, 1031-1032.

36. Steiner M, Steiner B, Wilhelm S, et al. (2000) Bone marrow transplantation and severe hypophosphataemia during hematopoietic reconstruction after allogeneic peripheral blood stem cell transplantation. Bone Marrow Transplant 25, 1015-1016.

37. Crook M, Swaminathan R \& Schey S (1996) Hypophosphataemia in patients undergoing bone marrow transplantation. Leuk Lymphoma 22, 335-337.

38. Raanani P, Levi I, Holzman F, et al. (2001) Engraftment-associated hypophosphatemia - the role of cytokine release and steep leukocyte rise post stem cell transplantation. Bone Marrow Transplant 27, 311-317. 
39. Wędrychowicz A, Goździk J, Krasowska-Kwiecień A, et al. (2006) Manifestation of toxocariasis in children with neuroblastoma treated with autologous hematopoietic transplants. Pediatr Hematol Oncol 23, 369-379.

40. Kagialis-Girard S, Mialou V, French M, et al. (2005) Thrombocytosis and toxocariasis: report of two pediatric cases. Pediatr Blood Cancer 44, 190-192.

41. Cetin T, Arpaci F, Dere Y, et al. (2002) Total parenteral nutrition delays platelet engraftment in patients who undergo autologous hematopoietic stem cell transplantation. Nutrition 18, 599-603.
42. Weisdorf SA, Lysne J, Wind D, et al. (1987) Positive effect of prophylactic total parental nutrition on long-term outcome of bone marrow transplantation. Transplantation 43, 833-838.

43. Mulder PO, Bouman JG, Gietema JA, et al. (1989) Hyperalimentation in autologous bone marrow transplantation for solid tumors. Comparison of total parental versus partial parental plus enteral nutrition. Cancer 64, 2045-2052.

44. Sheean PM \& Braunschweig CA (2007) Exploring the clinical characteristics of parenteral nutrition recipients admitted for initial hematopoietic stem cell transplantation. J Am Diet Assoc 107, 1389-1403. 